\title{
The Chromosomal Order of Genes Controlling the Major Histocompatibility Complex, Properdin Factor B, and Deficiency of the Second Component of Complement
}

\author{
Donald Raum, David Glass, C. B. Carpenter, Chester A. Alper, and \\ Peter H. SCHUR
}

From the Robert B. Brigham Hospital, Peter Bent Brigham Hospital, The Center for Blood Research, Harvard Medical School, Boston, Massachusetts 02120

A B S T RACT The relationship of the genes coding for HLA to those coding for properdin Factor B allotypes and for deficiency of the second component of complement (C2) was studied in families of patients with connective tissue disorders. Patients were selected because they were heterozygous or homozygous for C2 deficiency. 12 families with 15 matings informative for C2 deficiency were found. Of 57 informative meioses, two crossovers were noted between the C2 deficiency gene and the HLA-B gene, with a recombinant fraction of 0.035 . A lod score of 13 was calculated for linkage between C2 deficiency and HLA-B at a maximum likelihood value of the recombinant fraction of 0.04. 18 families with 21 informative matings for both properdin Factor B allotype and HLA-B were found. Of 72 informative meioses, three recombinants were found, giving a recombinant fraction of 0.042 . A lod score of 16 between HLA-B and Factor B allotypes was calculated at a maximum likelihood value of the recombinant fraction of 0.04 . A crossover was shown to have occurred between genes for Factor B and HLA-D, in which HLA-D segregated with HLA-A and B.

These studies suggest that the genes for Factor B and C2 deficiency are located outside those for HLA, that the order of genes is HLA-A, -B, -D, Factor B allotype, C2 deficiency, that the genes coding for C2 deficiency and Factor B allotypes are approximately 3-5 centimorgans from the HLA-A and HLA-B loci, and that the apparent lack of recombinants between the Factor B gene and C2 deficiency gene suggests

Dr. Carpenter is an Investigator of the Howard Hughes Medical Institute.

Received for publication 19 April 1976 and in revised form 2 August 1976. that these two genes lie in close proximity to one another.

\section{INTRODUCTION}

Evidence has been presented for the linkage of the HLA histocompatibility genes (HLA-A, B, C, D), ${ }^{1}$ collectively referred to as the major histocompatibility complex (MHC), to the gene for deficiency of the second component of complement (C2d) (2-4), and for the linkage of $\mathrm{MHC}$ to the gene for properdin Factor B allotype polymorphism (Bf) (5). A number of studies have presented data and attempted to map the order of the genes. Family studies by Teisberg et al. have suggested that the order of genes is HLA-A, B, Bf (6). A recombinant fraction (RF) between HLA and Bf of $6.1 \%$ has been observed, suggesting that they are, at most, 6 centimorgans apart (7). These data suggest that the Bf locus is outside the MHC. By contrast, studies by Lamm et al. and Hauptmann et al. suggest that Bf is between HLA-B and HLA-D $(8,9)$. The ability to

\footnotetext{
${ }^{1}$ Nomenclature and abbreviations used in this paper: The convention for denoting genetic traits follows the guidelines set up by the WHO-IUIS Terminology Committee (1) and is extended to include the properdin Factor B allotypes and C2 deficiency traits. The major histocompatibility complex (MHC) in man is called HLA and contains four recognizable loci: A (1st series, or LA); B (2nd series, FOUR); C (3rd series, AJ); and D (MLC, LD, MLR-S). A, B, and C are defined by serological techniques and $\mathrm{D}$ by mixed lymphocyte culture (MLC). Other abbreviations are C2d, C2 deficiency; C2, C2 normal levels; C4d, C4 deficiency; Bf, properdin Factor B; $\mathrm{RF}$, recombinant fraction; $\mathrm{S}, \mathrm{Bf}$ allotype slow; Sl, rare slow; $F$, fast; $F l$, rare fast; MLRF, maximum likelihood value of the recombinant fraction; PGM3, phosphoglucomutase-3; H-2, mouse histocompatibility locus; Ir, immune response gene; and $\mathrm{RR}$, relative response.
} 
define hereditary deficiency of C2d in the absence of homozygous deficient family members by the simultaneous use of immunochemical determinations, hemolytic assay and HLA gene complex association (4) has made it possible to survey a large number of unrelated patients and their families for heterozygous deficiency of the second component of complement. The aim of the present studies was to better define the linkage between the HLA, C2d, and $\mathrm{Bf}$ and to determine their order on the chromosome by studying large families and examining them for crossover events.

\section{METHODS}

HLA typing. HLA typing was done by the microdroplet lymphocyte cytotoxicity test (10) using antisera to define 36 specificities (antisera kindly provided by Dr. Donald Kayhoe, Transplantation and Immunology Branch, National Institute of Allergy and Infectious Diseases, Bethesda, Md.). Unrelated ethnically matched normal controls sequentially selected from the kidney donor population and a panel of normal individuals from the Tissue Typing Laboratory at the Peter Bent Brigham Hospital served as controls. The same antisera used in typing the patients and their families were used to type the controls.

Mixed lymphocyte culture. One way mixed lymphocyte cultures were performed as a microlymphocyte transformation test (11) as described in detail previously (4).

Complement component measurements. Serum protein concentrations of $\mathrm{C} 2, \mathrm{C} 4, \mathrm{C} 6$, and other complement components were measured by radial immunodiffusion using monospecific antisera developed in this laboratory (12-14). Properdin Factor B allotypes were recognized as described previously (15). Bf allotypes are denoted as Bf S (slow), Bf Sl (rare slow), Bf F (fast), or Bf Fl (rare fast). The frequency of $\mathrm{Bf} S$ in the general population studied is about 0.7 . Hemolytic C2 was measured by a modification of the method of Borsos and Rapp $(16,17)$. Heterozygous C2 deficiency in the absence of homozygous deficient individuals was recognized as previously described (4).

Calculation of the log of the odds (lod score) for the linkage of two genes and the maximum likelihood value of the recombinant fraction. The $\mathrm{RF}$ is defined as the number of observed crossovers divided by the number of observed meioses. Conceptually, the RF is the probability that two genes, one at each locus, are not passed on together to an offspring Lod scores were calculated by the method of Morton (18) and as modified by Maynard-Smith et al. (19) and Smith (20). Initially, all lod scores were computed for double-back crosses (matings in which one parent was doubly heterozygous and the other was at least singly homozygous at one of the two loci scored) between C2 deficiency and HLA, and between properdin Factor b allotype and HLA with no assumption as to phase (whether in a given person, specific HLA and Bf genes are on the same or different chromosomes). The likelihood value of the recombinant fraction $=\theta$. Since these genetic loci were already known to be located on the sixth chromosome near the HLA region, it was felt to be valid to calculate these lod scores as if phase were known unless the number of progeny observed was two and there was one recombinant and one nonrecombinant. In fact, the calculation of the lod score in this case is identical for both phase known and phase unknown. The maximum likelihood value of the recombinant fraction
TABLE I

Lod scores: Bf and C2d vs. HLA

Bf

$\mathrm{C} 2 \mathrm{~d}$

\begin{tabular}{lcc} 
Kindred & 18 & 12 \\
Informative matings & 21 & 15 \\
Informative meioses & 72 & 57 \\
Recombinants & 3 & 2 \\
Recombinant fraction & 0.042 & 0.035 \\
Lods at $\theta=0.001$ & 12.64 & 11.74 \\
0.010 & 15.36 & 12.92 \\
0.020 & 15.99 & 13.28 \\
0.030 & 16.21 & 13.39 \\
0.040 & 16.27 & 13.39 \\
0.050 & 16.25 & 13.34 \\
0.100 & 15.50 & 12.56 \\
0.200 & 12.88 & 10.21 \\
0.450 & 2.69 & 2.12 \\
\hline
\end{tabular}

(MLRF) was taken as that $\theta$ which gave the greatest lod score. No evidence has been presented for differences in the crossover rate in males and females in the MHC region and therefore no correction has been made for this. Theoretical justification for the use of lod scores for the determination of the order of loci on a chromosome is given by Sturt (21).

Estimation of linkage disequilibrium. The detection of linkage disequilibrium was done by the methods of Bodmer $(22,23)$, and Kojima and Lewontin (24).

Population studied. During the course of studies of ac quired abnormalities of the complement system in patients with rheumatic diseases (25), a number of individuals were encountered who appeared to be heterozygous deficient for C2 (4). Families were studied where propositi were previously shown to have complement abnormalities $(4,26)$.

\section{RESULTS}

18 kindred were studied which were informative (one parent doubly heterozygous) for the linkage between properdin Factor B allotypes and HLA. Of the 18 kindred, there were 21 informative matings with 72 informative meioses. In this way, three recombinant events, described below, were noted in the offspring. The RF is $\mathbf{0 . 0 4 2}$. The lod scores for these matings were computed at likelihood values of the recombinant fraction $(\theta)$ from 0.001 to 0.45 . The lod score was 16.27 at the MLRF $=0.04$.

12 kindred were studied which were informative for the linkage of C2 deficiency and HLA. Of the 12 kindred, there were 15 informative matings with 57 informative meioses. Two recombinant events, described below, were noted in the offspring. The RF is 0.035 . The lod scores were computed and the score was 13.39 at the MLRF $=0.04$. These data are presented in Table I. In all kindred studied there were no recombinants observed between HLA-A and B loci, in keeping with the much lower RF (less than 0.01) known to exist for these loci. 


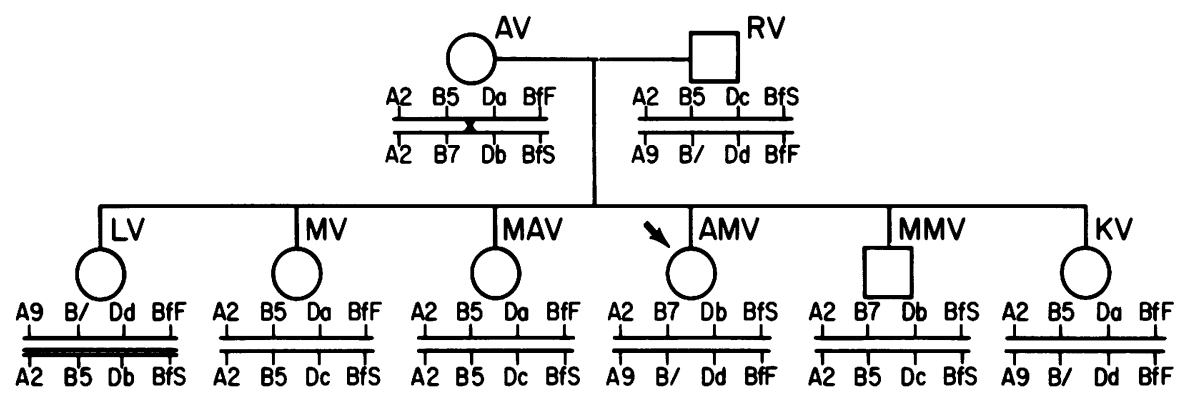

FIGURE 1 In a pedigree, males are indicated by squares, females by circles. The propositus is indicated by an arrow. Each individual is identified by initials written to the right of the symbol and below each symbol is given the deduced genotypes of HLA-A, HLA-B, Bf, and C2d locus. HLA specificities are as given in Footnote 1 . Bf allotypes are denoted as S (slow), F (fast), Fl (rare fast), or Sl (rare slow). HLA-D alleles are given the arbitrary designation a, b, c, or d. A genome denoted by a double line contains a recombination. Probable sites of recombination are denoted by solid $\mathrm{X}$ and possible alternative sites of recombination are denoted by dotted $\mathrm{X}$.

Families in which crossover events were detected for either properdin Factor B or C2d were examined in detail. Fig. 1 presents family $\mathrm{V}$, in which the product of a recombinant for properdin Factor B allotypes has been observed in LV. The phases of $\mathrm{Bf}$ allotypes in $\mathrm{AV}$ and $\mathrm{RV}$ are known from the study of HLA and Bf phenotypes of their parents. Therefore there are a maximum of five children who have received their respective HLA genotypes and $\mathrm{Bf}$ allotypes from their parents without a recombinant event between the loci during either parental meiosis. These children are MV, MAV, MMV, KV, and the propositus, AMV. Two-way mixed lymphocyte cultures (MLCs) were performed between siblings and parents.
$\mathrm{AV}$ and RV are mutually stimulatory with each of their children. LV is mutually stimulatory with siblings MAV and KV and nonstimulatory with AMV. Therefore, the recombinant event must have occurred in AV between HLA-B and HLA-D. This recombinant event places the gene for Bf allotypes on the HLA-D side of HLA-B.

Fig. 2 presents family $R$ in which a recombinant for both Factor B and C2 deficiency has been observed, and has also been noted previously (4). The phase of C2 deficiency, HLA, and Factor B allotypes is established by the study of siblings, nieces, and nephews of JaRa. This establishes the child JuRa to have a recombinant genome A2, B5,

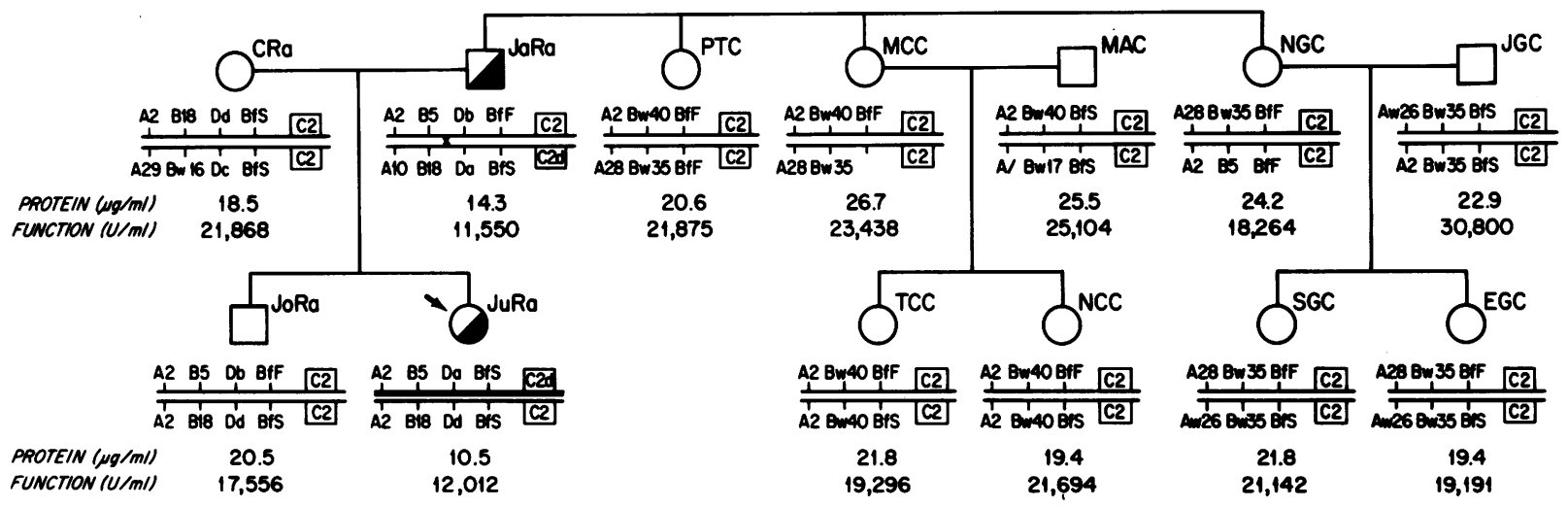

FIGURE 2 In a pedigree, males are indicated by squares, females by circles. Heterozygotes are shown by half-blackened symbols and the propositus is indicated by an arrow. Each individual is identified by initials written to the right of the symbol and below each symbol is given the deduced genotypes for HLA-A, HLA-B, Bf, and C2d locus. HLA specificities are as given in Footnote 1. Bf allotypes are denoted as S (slow), F (fast), Fl (rare fast), or Sl (rare slow) and C2 level denoted as C2d for deficient or C2 for normal. Where applicable both C2 protein and functional levels are given. HLA-D alleles are given the arbitrary designation $a, b$, c, or d. A genome denoted by a double line contains a recombination. Probable sites of recombination denoted by solid $\mathrm{X}$ and possible alternative sites of recombination denoted by dotted $\mathrm{X}$. 


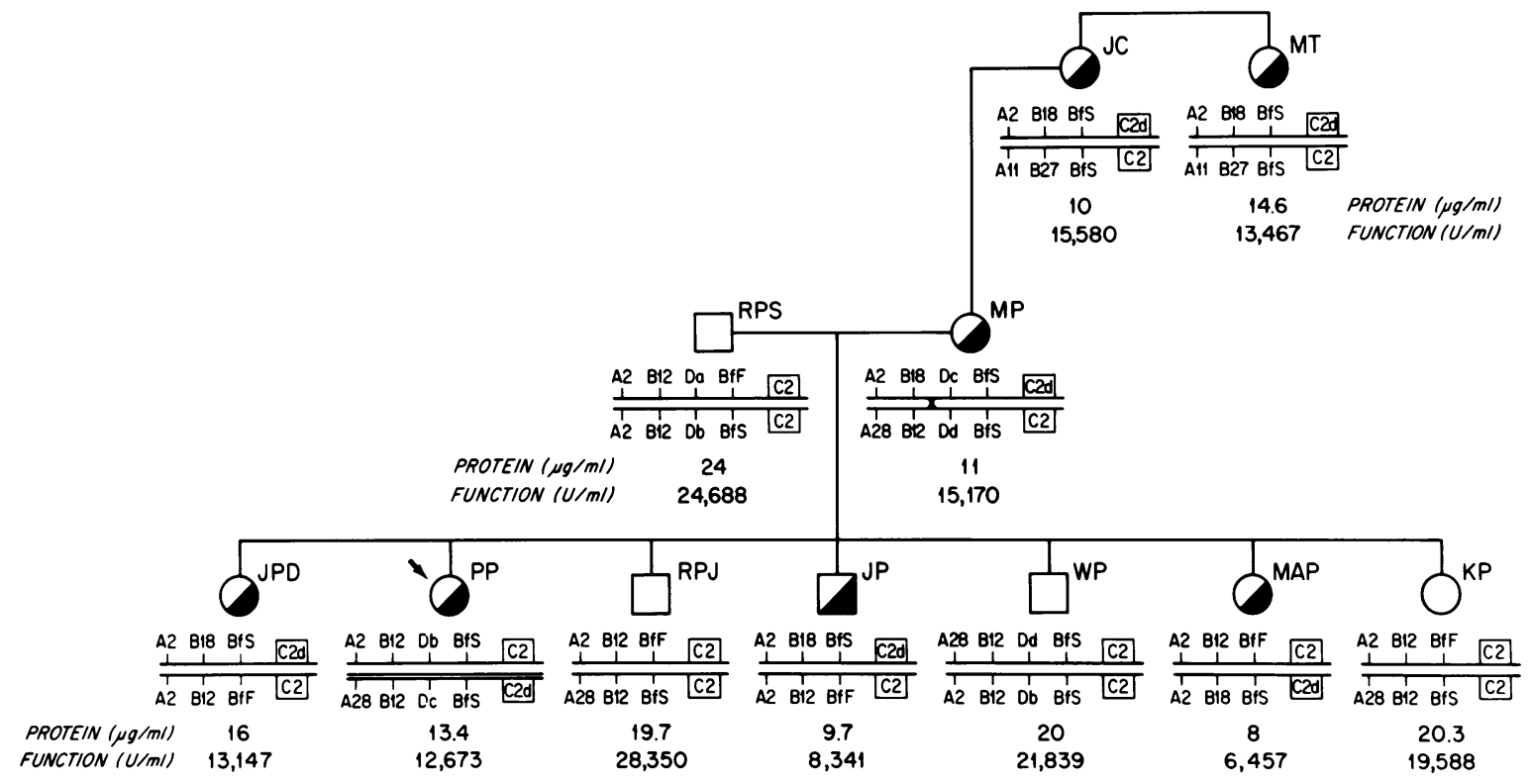

FIGURE 3 In a pedigree, males are indicated by squares, females by circles. Heterozygotes are shown by half-blackened symbols and the propositus is indicated by an arrow. Each individual is identified by initials written to the right of the symbol and below each symbol is given the deduced genotypes for HLA-A, HLA-B, Bf, and C2d locus. HLA specificities are as given in Footnote 1. Bf allotypes are denoted as S (slow), F (fast), Fl (rare fast), or Sl (rare slow) and C2 level denoted as C2d for deficient or C2 for normal. Where applicable both $\mathrm{C} 2$ protein and functional levels are given. HLA-D alleles are given the arbitrary designation a, b, c, or d. A genome denoted by a double line contains a recombination. Probable sites of recombination denoted by solid $\mathrm{X}$ and possible alternative sites of recombination denoted by dotted $\mathrm{X}$.

Bf S, C2d from her father. The mutual stimulation of her HLA-identical sibling JoRa places the genes for $\mathrm{Bf}$ and C2d on the HLA-D side of HLA-B.

Fig. 3 presents family $P$ in which a recombinant for $\mathrm{C} 2 \mathrm{~d}$ has been observed. The phase of C2 deficiency and HLA is established by the study of three generations. The genotype of the mother, MP, is deduced from her mother, JC. The genome of the father, RPS, is deduced in a manner to reduce the number of observed recombinants. If this is the case, then the child PP, who is the propositus with juvenile rheumatoid arthritis, received the genome $\mathrm{A} 2, \mathrm{~B} 12$, Bf S from her father and genome with recombinant event A28, B12, Bf S, C2d from her mother. Mutual stimulation of mother, MP, and PP; mother and WP, an HLA identical sibling of PP; and of PP and WP suggests that the recombinant event occurred between HLA-B and HLA-D and also places the gene for C2d on the HLA-D side of HLA-B.

Fig. 4 presents family $B$ in which a recombinant for Factor B allotypes has been observed. C2 deficiency has been assumed to be in coupling (i.e., on the same chromosome) with Al0 because of their known linkage disequilibrium (2-4). The phase of Factor B allotypes in JB was assumed to minimize the number of observed recombinants, as would be ex- pected for two closely linked genes. In this manner the child PB, who was the juvenile rheumatoid arthritis propositus, is thought to have received the genome $\mathrm{A} 10, \mathrm{Bw} 40, \mathrm{Bf} \mathrm{S}, \mathrm{C} 2 \mathrm{~d}$ from her father and the recombinant genome $\mathrm{A} 2, \mathrm{Bw} 15, \mathrm{Bf} \mathrm{S}$ from her mother. All five members of this family have been studied with two way MLC reactions and all combinations are strongly stimulatory. The relative responses are given in Table II and are approximately 1.0 for every combination. It was expected that $\mathrm{DB}$ and $\mathrm{PB}$ would not stimulate one another, but $\mathrm{DB}$ and $\mathrm{PB}$ have a relative response $(\mathrm{RR})=1.55$ compared to $\mathrm{JB}$ when the stimulatory (mitomycin-treated) cell is $\mathrm{DB}$; and $\mathrm{RR}=1.06$ when compared to WB. $\mathrm{DB}$ and $\mathrm{PB}$ have an $\mathrm{RR}=0.84$ compared to JB when the stimulatory cell is $P B$, and $R R=0.80$ when compared to WB. Therefore, the crossover during paternal meiosis must have occurred outside of the group of genes delimited by the loci for HLA-A, B, and D. This establishes the order of genes as HLA-A, B, D, Bf.

A tabulation of the frequency of the association of alleles at the loci studies was done and is presented in Table III. Of the population studied, 57 members with C2 deficiency (including 1 homozygous deficient) had Factor B allotypes determined. In all 58 cases the C2 deficiency could be deduced to be linked to $\mathrm{Bf} S$. 


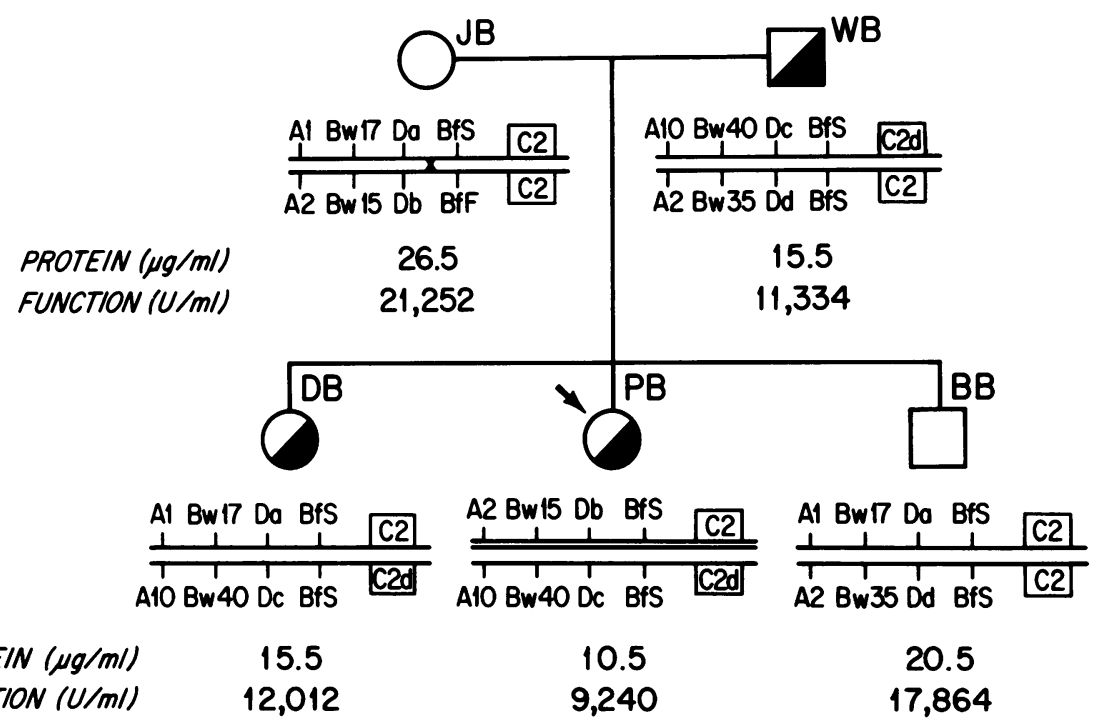

FIGURE 4 In a pedigree, males are indicated by squares, females by circles. Heterozygotes are shown by half-blackened symbols and the propositus is indicated by an arrow. Each individual is identified by initials written to the right of the symbol and below each symbol is given the deduced genotypes for HLA-A, HLA-B, Bf, and C2d locus. HLA specificities are as given in Footnote 1. Bf allotypes are denoted as S (slow), F (fast), Fl (rare fast), or Sl (rare slow) and C2 level denoted as $\mathrm{C} 2 \mathrm{~d}$ for deficient or $\mathrm{C} 2$ for normal. Where applicable both $\mathrm{C} 2$ protein and functional levels are given. HLA-D alleles are given the arbitrary designation a, b, c, or d. A genome denoted by a double line contains a recombination. Probable sites of recombination denoted by solid $\mathrm{X}$ and possible alternative sites of recombination denoted by dotted $\mathrm{X}$.

Of these 58 genomes with $\mathrm{C} 2 \mathrm{~d}$ and $\mathrm{Bf} \mathrm{S}, 10$ were linked to $\mathrm{B} 18$ (but not $\mathrm{A} 10$ or closely related Aw25), 3 were linked to A10 or Aw25 (but not B18), and 43 were linked to both A10 or Aw25 and B18. Of the two which were linked to neither A10 (Aw25) nor B18, one was shown to be a result of a crossover in which the $\mathrm{C} 2 \mathrm{~d}$ and $\mathrm{Bf} \mathrm{S}$ had been linked with $\mathrm{B} 18$ in the mother. In the second case, in a C2 homozygous deficient individual, the genotype was A10, B18, S,

TABLE II

MLC Response of Family B*

\begin{tabular}{|c|c|c|c|c|}
\hline $\begin{array}{l}\text { Responding } \\
\text { cell (R) }\end{array}$ & $\begin{array}{l}\text { Stimulating } \\
\text { cell }(\mathbf{S})\end{array}$ & $\begin{array}{l}\text { Comparison } \\
\text { cell }\end{array}$ & $\begin{array}{l}\text { Relationship } \\
\quad(\mathrm{R} \text { to } \mathrm{S})\end{array}$ & $\begin{array}{l}R R \text { for } \\
R \text { to } S\end{array}$ \\
\hline PB & WB & JB & Propositus-Father & 1.0 \\
\hline DB & WB & $\mathrm{JB}$ & Sibling-Father & 1.01 \\
\hline BB & WB & JB & Sibling-Father & 0.76 \\
\hline $\begin{array}{c}\text { Unrelated } \\
\text { control }\end{array}$ & WB & JB & Control-Father & 0.76 \\
\hline PB & DB & JB & Propositus-Sibling & 1.55 \\
\hline DB & PB & JB & Sibling-Propositus & 0.84 \\
\hline PB & DB & WB & Propositus-Sibling & 1.06 \\
\hline PB & DB & WB & Sibling-Propositus & 0.80 \\
\hline
\end{tabular}

All possible combinations of five family members and unrelated control give similar results.

* For genotype refer to Fig. 5.
$\mathrm{C} 2 \mathrm{~d} / \mathrm{Al}, \mathrm{B} 8, \mathrm{~S}, \mathrm{C} 2 \mathrm{~d}$ in whom the father was not available but the phase of A10, B18, S, C2d could be established in the mother through a second marriage and the offspring of that mating. Of 58 known C2d and $\mathrm{Bf}$ genomes there is a $100 \%$ association of $\mathrm{C} 2 \mathrm{~d}$ with $\mathrm{Bf} \mathrm{S}$, a $91 \%$ association of $\mathrm{C} 2 \mathrm{~d}$ with $\mathrm{B} 18$ (10 plus 43 of 58), and a $79 \%$ association of C2d with A10 or Aw25 ( 3 plus 43 of 58 ).

An estimate of linkage disequilibrium is given by a

TABLE III

Frequency of C2d Association with Bf and HLA

\begin{tabular}{lcr}
\hline \multicolumn{1}{c}{ Genotype } & $\begin{array}{c}\text { Observed number of } \\
\text { genomes linked to } \\
\text { C2d }\end{array}$ & $\begin{array}{c}\text { Percent } \\
\text { observed } \\
58\end{array}$ \\
\hline & 58 & $\%$ \\
C2d-S & 0 & 100 \\
C2d-F & 10 & 0 \\
$\begin{array}{l}\text { C2d-B18 } \\
\text { but not A10 or Aw25 }\end{array}$ & 3 & 17 \\
$\begin{array}{l}\text { C2d-A10 or Aw25 } \\
\text { but not B18 }\end{array}$ & 43 & 5 \\
$\begin{array}{l}\text { C2d-B18-A10-Aw25 } \\
\text { C2d but neither } \\
\text { B18 nor A10 or Aw25 }\end{array}$ & 2 & 74 \\
\hline
\end{tabular}


delta value. A delta value is an estimate of the difference between the expected and observed association of alleles at closely linked loci. For most alleles delta is about 0.001 and is significant if greater than 0.01 . For the two-locus model of linkage disequilibrium the delta value for C2d with A10 (or Aw25) and B18 is approximately 0.2 in our population. Since the number of loci studied (HLA-A, B, D, Bf, C2d) in some families was five and the loci are probably located within 5 centimorgans of one another, a more complex model of linkage disequilibrium may be applied which suggests the actual delta value for these five alleles to be a minimum of 0.22 .

Of the families studied for C2 deficiency and Factor $B$ allotypes, in 15 the parents and siblings of the proband were also studied. This was the case of all four families in which crossover events were observed in the region studied. Of the 16 probands, all had juvenile rheumatoid arthritis or systemic lupus erythematosus; three were shown to have had crossovers in the chromosome region studied, as demonstrated in Figs. 2,3 , and 4 . Of the 38 siblings studied, all of whom are well, only one other crossover event was observed. Since meioses were counted for both Bf and C2d there were four crossovers observed in 27 meioses for probands and one crossover observed in 64 meioses for well siblings. The $\chi^{2}=6.42, P=0.01$.

\section{DISCUSSION}

The MHC has been assigned to chromosome 6 by the observation of a pericentric inversion (27). The orientation of the HLA loci in respect to the centromere has been established by study of phosphoglucomutase-3. Phosphoglucomutase-3 (PGM3) has been assigned to chromosome 6 by somatic cell hybrid studies (28). The orientation of the HLA region with regard to PGM3 has been established by studies of crossovers within the HLA region (29). PGM3 is thought to be linked to the centromere of chromosome 6 from studies of cultures of ovarian teratomas (30). These data give the order of genes as: Centromere . . . PGM3, HLA-D, B, C, A. The RF between HLA-D and HLA-B has been estimated and these loci are less than 1 centimorgan apart (31). The HLA-A and HLA-B loci are thought to be at most 1 centimorgan apart (32).

Several investigators have previously reported data concerning the linkage of $\mathrm{Bf}$ to the MHC. Allen (5) described 12 families with 44 informative children. No recombinants were found and the lod score was approximately 10. Rittner et al. (7) studied 21 families with 82 informative meioses: of these, 5 suggested a single recombinant event between HLA and Bf. From this they calculated an RF of 0.061 . A lod score of 10 was calculated at $\theta=0.1$. Of the five crossovers, three apparently were maternal and two were paternal; however, no difference was seen in the lods scored separately from the maternal and paternal meioses. This is unlike the data observed for HLA and PGM3, which suggests different rates of crossing over in the male and female in the PGM3 region $(33,34)$. In the rhesus monkey linkage of $\mathrm{Bf}$ to the histocompatibility loci has been demonstrated with MLRF of 0.02 and lod score 15 (35), suggesting a similar relationship of these loci in man and monkey.

Teisberg et al. (6) studied three families known to have a crossover between the HLA-A and B, and demonstrated the segregation of $\mathrm{Bf}$ and HLA-B. They studied 23 matings with 90 informative offspring in whom no recombinants have been seen. They have studied the linkage of the HLA region to the $\mathrm{Bf}$ locus in a slightly different manner. They have scored separately for HLA-A alleles and Bf allotypes and HLA-B alleles and Bf allotypes. 17 kindred with $49 \mathrm{Bf} / \mathrm{HLA}$ informative matings and 178 children were studied. Of these 178 meioses, three recombinants were observed. This is an RF of 0.017 . In our data, we have observed three HLA/Bf recombinants in 72 informative meioses, to give the $\mathrm{RF}$ 0.042 . If one sums all the previous data, 466 informative meioses with 11 recombinants for HLA-Bf have been observed, giving an RF of 0.024 . If one accepts, as discussed later, that our data may inadvertently have selected for crossovers by the examination of a disease population, as did Rittner et al., then one could accept that in a general population the RF may actually be somewhat smaller. Of the crossovers observed by Rittner et al., those reported (7) do not unequivocally demonstrate the location of $\mathrm{Bf}$ with regard to the HLA-D; but in a footnote, they suggest that the typing of two families revealed a crossover between the HLA-D and Bf, in which $B f$ segregates from HLA-B and D. Lamm et al. report a recombination between HLA-D and HLA-B in which Bf follows HLA-B (8). Similar data has been presented by Hauptmann et al. (9). In our data, we report a crossover between HLA-D and Bf, in which Bf segregates from HLA-B and $D$, and definitively places Bf between PGM3 and HLA-D. It is difficult to explain these conflicting results, but assuming that both sets of observations are correct, they lead one to invoke the possibility that Bf may exist in some families as redundant loci (36).

We have observed no recombinant event between $\mathrm{C} 2 \mathrm{~d}$ and Bf. In addition, a marked linkage disequilibrium between HLA-A10, B18, Dw2, and C2 deficiency has been noted (37). Our data concerning the linkage of HLA to the locus coding for C2d indicates, both by RF and by maximum likelihood estimate of the RF, that this locus is very near to the Bf locus. Our estimate of MLRF by lod scores for C2d and HLA is in agreement with that reported for 


\section{CHROMOSOME 6}

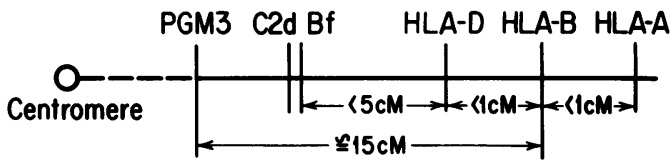

FIgURE 5 Proposed chromosome map distances for the loci discussed. Centromere denoted by a circle. The linear arrangement of genes is denoted by vertical lines and abbreviated as PGM3, deficiency of the second component of complement (C2d), properdin Factor B allotypes (Bf), and histocompatibility loci (HLA-A, B, D). Their approximate distances in centimorgans $(\mathrm{cM})$ are given between the vertical lines.

families with known C2d homozygotes (38). This linkage disequilibrium and the absence of observed recombinants between these loci may be explained by the existence of a human "T-locus" like that associated with the H-2 locus of the mouse (39). This locus is about 8 centimorgans from the $\mathrm{H}-2$ on the centromere side of mouse chromosome 17 . That fact that wild mice are polymorphic for t-alleles, and that the MHC of the mouse lies within the area of crossover suppression associated with recessive lethal T-locus mutants, suggests in mice that the T-locus may serve to maintain this chromosome region as a "super-gene" complex. Our data also suggest an alternative explanation. When the genotypes were deduced, C2d was associated with the B18 alleles in approximately $91 \%$ of cases, and C2d was associated with the sum of A10 and Aw25 alleles in approximately $79 \%$ of cases. The decreasing association of alleles as genetic distance between loci increases could be explained by recent mutation at one locus with not enough time for the various alleles at several loci to reach the equilibrium predicted by the Hardy-Weinberg law. That this is the case is also supported by the observation of $\mathrm{C} 2 \mathrm{~d}$ association with A3, B5 and Aw30, B13 haplotypes (40).

No recombinant event has been observed between HLA-D and C2d which would definitively place C2d relative to the positions of HLA-A, B, and D; but our data suggest that $\mathrm{C} 2 \mathrm{~d}$ is, like $\mathrm{Bf}$, located outside the major histocompatibility complex, and that $\mathrm{C} 2 \mathrm{~d}$ arose as a relatively recent mutation; the propositus carrying the A10, B18, Bf S, C2d genotype. Friend et al. have presented evidence locating the $\mathrm{C} 2 \mathrm{~d}$ locus between HLA-D and PGM3 (41).

Of the 54 children for whom both parents were known, including 16 propositi with known juvenile rheumatoid arthritis or systemic lupus erythematosus, three of the crossover events were observed in propositi, while only one was observed in a normal sibling. This suggests that a crossover during meiosis may play some part in the pathophysiology of these diseases. Of the 13 propositi in whom no crossover was observed, meiosis was informative in only one parent and therefore a crossover is not ruled out in these children. An increase in intra-HLA recombinants has been observed in juvenile diabetes mellitus (42). Relevant to this is a recent observation by Dorf et al. (43) that complementation may occur between immune response (Ir) genes. Further, it appears that an immune response may occur when both Ir genes are in the cis position (i.e., on the same chromosome), but not in the trans configuration of the $F_{1}$ hybrid (i.e., each gene on separate chromosomes). It is also suggested that cis effects have helped maintain close linkage of Ir genes during mammalian evolution (44). The increased incidence of crossovers with the loss of one or more complementing Ir genes among this rheumatic disease population may account in part for some of their immunological abnormalities.

It has also been demonstrated in the mouse that complement level correlates with SsSlp genotype, which is part of the mouse MHC, and not with $\mathrm{H}-2 \mathrm{~K}$, H-2D, or Ir genotypes (45). It has been subsequently shown that the Ss protein is a homologue of human C4 (46-48). In addition, evidence has been presented that $\mathrm{C} 3$ level during ontogeny is MHC-linked in mouse (49). The mapping of C2d, C4d, and Bf near the MHC suggests that this region may be of importance in inflammatory processes.

Though it is not possible at this time to ascertain the significance of the observed linkage disequilibrium between alleles at loci located over a distance of about 5 centimorgans, it is concluded that recent mutation is the most likely cause of the observed disequilibrium of HLA and C2d. It is concluded that Bf lies between PGM3 and HLA-D. The lack of recombinants between $\mathrm{Bf}$ and C2d and similar MLRFs suggests they lie in proximity to one another and hence that $\mathrm{C} 2 \mathrm{~d}$ lies between PGM3 and HLA-D. Fig. 5 summarizes these relationships. It is hypothesized that the close proximity of the genes coding for $\mathrm{C} 2 \mathrm{~d}$ and $\mathrm{Bf}$ through their similar functional positions, respectively, in the classical and alternate pathways suggests the possibility that these two proteins arose by gene duplication and divergent evolution, and from other data, that the $\mathrm{Bf}$ gene and alternate pathway were prototypes. The association of disease with crossover events suggests that connective tissue disease may be pathophysiologically related to the disruption of cis-Ir gene complementation. If this association with connective tissue disease proves to be correct, $\mathrm{Bf}$ and $\mathrm{C} 2 \mathrm{~d}$ are probably closer to HLA-B in a normal population than suggested by the RF of 0.035 and 0.042 .

\section{ACKNOWLEDGMENTS}

We gratefully acknowledge the excellent assistance of Rosanne Stein, Eugenia Holbrook, Gerri Schulman, Margaret Stas- 
sen, and the physicians of the Robert B. Brigham Hospital who made patients available for study. Special acknowledgment is made to the many families who gave willingly of their time and blood.

Supported, in part, by grants AM 11414, AI 00366, AM 05577, AM 13855, RR 05669, and Am 5076 from the U.S. Public Health Service, the Easter Seal Society, the New England Peabody Foundation, the Histocompatibility Research Fund of the Peter Bent Brigham Hospital, and the Charles King, M. L. Riley, and Schrafft Charitable Trusts.

\section{REFERENCES}

1. D. B. Amos, chairman. 1976. WHO-IUIS Terminology Committee. Nomenclature for factors of the HL-A system. Transplant. Proc. 8: 109-114.

2. Fu, S. M., H. G. Kunkel, H. P. Brusman, F. H. Allen, Jr., and M. Fotino. 1974. Evidence for linkage between HL-A histocompatibility genes and those involved in the synthesis of the second component of complement. J. Exp. Med. 140: 1108-1111.

3. Day, N. K., P L'Esperance, R. A. Good, A. F. Michael, J. A. Hansen, B. Dupont, and C. Jersild. 1975. Hereditary C2 deficiency: genetic studies and association with the HL-A system. J. Exp. Med. 141: 1464-1469.

4. Gibson, D. J., D. Glass, C. B. Carpenter, and P. H. Schur. 1976. Hereditary C2 deficiency: diagnosis and HLA gene complex associations. J. Immunol. 116: 1065-1070.

5. Allen, F. H., Jr. 1974. Linkage of HL-A and GBG. Vox Sang. 27: $382-384$

6. Teisberg, P., B. Olaisen, T. Gedde-Dahl, Jr., and E. Thorsby. 1975. On the localization of the $G b$ locus within the MHS region of chromosome no. 6. Tissue Antigens. 5: 257-261.

7. Rittner, Ch., H. Grosse-Wilde, B. Rittner, B. Netzel, S. Scholz, H. Lorenz, and E. D. Albert. 1975. Linkage group HL-A-MLC-Bf (properdin factor B). The site of the $\mathrm{Bf}$ locus at the immunogenetic linkage group on chromosome 6. Humangenetik. 27: 173-183.

8. Lamm, L. U., F. Jørgensen, and F. Kissmeyer-Nielsen. 1976. Bf maps between HLA-A and D loci. Tissue Antigens. 7: 122-124.

9. Hauptmann, G., M. Sasportes, M. M. Tongio, S. Mayer, and J. Dausset. 1976. The localization of the Bf locus within the MHS region on chromosome no. 6. Tissue Antigens. 7: 52-54.

10. Mittal, K. K., M. R. Mickey, D. P. Singal, and P. I Terasaki. 1968. Serotyping for homotransplantation. XVIII. Refinement of microdroplet lymphocyte cytotoxicity test. Transplantation (Baltimore). 6: 913-927.

11. Hartzman, R. J., M. Segall, M. L. Bach, and F. H. Bach 1971. Histocompatibility matching. VI. Miniaturization of the mixed leukocyte culture test: a preliminary report. Transplantation (Baltimore). 11: 268.

12. Schur, P. H., A. Connelly, and T. C. Jones. 1975. Phylogeny of complement components in non-human primates. J. Immunol. 114: 270-273.

13. Fearon, D. T., S. Ruddy, P. H. Schur, and W. R. McCabe. 1975. Activation of the properdin pathway of complement in patients with gram-negative bacteremia. N. Engl. J. Med. 292: 937-940.

14. Alpert, E., M. Monroe, and P. H. Schur. 1970. A method for increasing the sensitivity of radial-immunodiffusion assay. Lancet. 1: 1120.

15. Alper, C. A., T. Boenisch, and L. Watson. 1972 Genetic polymorphism in human glycine-rich betaglycoprotein. J. Exp. Med. 135: 68-80.
16. Ruddy, S., M. R. Klemperer, F. S. Rosen, K. F. Austen and J. Kumate. 1970. Hereditary deficiency of the second component of complement $(\mathrm{C} 2)$ in man: correlation of C2 hemolytic activity with immunochemical measurements of C2 protein. Immunology. 18: 943-954.

17. Borsos, T., and H. J. Rapp. 1967. Immune hemolysis: a simplified method for the preparation of EAC4 with guinea pig or with human complement. J. Immunol. 99: $263-268$.

18. Morton, N. E. 1955. Sequential tests for the detection of linkage. Am. J. Hum. Genet. 7: 277-318.

19. Maynard-Smith, S., L. S. Penrose, and C. A. B. Smith. 1962. Mathematical Tables for Research Workers in Human Genetics. Little, Brown and Co., Boston. 74 pp.

20. Smith, C. A. B. 1959. Some comments on the statistical methods used in linkage investigations. Am. J. Hum. Genet. 11: 289-304.

21. Sturt, R. 1975. The use of lod scores for the determination of the order of loci on a chromosome. Ann. Hum. Genet. 39: 255-260.

22. Cavalli-Sforza, L. L., and W. F. Bodmer. 1971. The Genetics of Human Populations. W. H. Freeman and Co. Publishers, San Francisco. 247-248 and 283-288.

23. Bodmer, W. F. 1973. Histocompatibility antigens. Part 2. Genetics of the HL-A and H-2 major histocompatibility systems. In Defense and Recognition, Biochemistry Series 1, Volume 10. R. R. Porter, volume editor. University Park Press, Baltimore. 295-328.

24. Kojima, K., and R. C. Lewontin. 1970. Evolutionary significance of linkage and epistaxis. In Mathematical Topics in Population Genetics. Ken-ichi Kojima, editor. Springer-Verlag New York Inc., New York. 367-388.

25. Schur, P. H. 1975. Complement in lupus. In Clinics in Rheumatic Diseases, Systemic Lupus Erythematosus. Naomi S. Rothfield, editor. Volume 1. W. B. Saunders Co. Ltd., London. 519-543.

26. Glass, D., D. Raum, J. S. Stillman, and P. H. Schur. 1976. Inherited deficiency of the second component of complement. Rheumatic disease associations. $J$. Clin. Invest. 58: 853-861.

27. Lamm, L. U., U. Friedrich, G. B. Petersen, J. Jørgensen, J. Nielsen, A. J. Therkelsen, and F. Kissmeyer-Nielsen. 1974. Assignment of the major histocompatibility complex to chromosome no. 6 in a family with a pericentric inversion. Hum. Hered. 24: 273-284

28. Jongsma, A., H. van Someren, A. Westerveld, A. Hagemeijer, and P. Pearson. 1973. Localization of genes on human chromosomes by studies of human-Chinese hamster somatic cell hybrids. Assignment of PGMs to chromosome $\mathrm{C} 6$ and regional mapping of the PGD, PGM, and pep-C genes on chromosome $\mathrm{A}_{1}$. Humangenetik. 20: 195-202.

29. Lamm, L. U., F. Kissmeyer-Nielsen, A. Svejgaard, G. B. Petersen, E. Thorsby, W. Mayr, and C. Högman. 1972. On the orientation of the HL-A region and the PGM 3 locus in the chromosome. Tissue Antigens. 2: 205-214.

30. Ott, J., F. Hecht, D. Linder, E. W. Lovrien, and B. Kaiser-McCaw. 1975. Human centromere mapping using teratoma data. Third International Workshops on Human Gene Mapping. In press.

31. Keuning, J. J., J. G. van den Tweel, B. W. Gabb, A. Termijtelen, E. Goulmy, E. Blokland, B. G. Elferink and J. J. van Rood. 1975. An estimation of the recombination fraction between the MLC locus and the FOUR locus. Tissue Antigens. 6: 107-115.

32. Bodmer, W. F., J. G. Bodmer, and M. Tripp. 1970 Recombination between the LA and 4 loci of the HL-A 
system. In Histocompatibility Testing. A. S. Munksgaard, Copenhagen. 187-191.

33. Lamm, L. U., I-L. Thorsen, G. B. Petersen, J. Jørgensen, K. Henningsen, B. Bech, and F. Kissmeyer-Nielsen. 1975. Data on the HL-A linkage group. Ann. Hum. Genet. 38: 383-390.

34. Weitkamp, L. R., J. J. van Rood, E. Thorsby, W. Bias, M. Fotino, S. D. Lawler, J. Dausset, W. R. Mayr, J. Bodmer, F. E. Ward, J. Seignalet, R. Payne, F. KissmeyerNielsen, R. A. Gatti, J. A. Sachs, and L. U. Lamm. 1973. The relation of parental sex and age to recombination in the HL-A system. Hum. Hered. 23: 197-205.

35. Ziegler, J. B., C. A. Alper, and H. Balner. 1975. Properdin factor B and histocompatibility loci linked in the rhesus monkey. Nature (Lond.). 254: 609-611.

36. Tartof, K. D. 1975. Redundant genes. Ann. Rev. Genet. 9: 355-385.

37. Fu, S. M., R. Stern, H. G. Kunkel, B. Dupont, J. A. Hansen, N. K. Day, R. A. Good, C. Jersild, and M. Fotino. 1975. Mixed lymphocyte culture determinants and $C_{2}$ deficiency: LD-7a associated with $\mathrm{C} 2$ deficiency in four families. J. Exp. Med. 142: 495-506.

38. Alper, C. A., and R. S. Rosen. 1976. Genetics of the complement system. Adv. Hum. Genet. In press.

39. Bennett, D. 1975. The T-locus of the mouse. Cell. 6: 441-454.

40. Wolski, K. P., F. R. Schmid, and K. K. Mittal. 1976. Genetic linkage between the HL-A system and a deficit of the second component (C2) of complement in four generations of a family. Tissue Antigens. 7: 35-38.

41. Friend, P. S., B. S. Handwerger, Y. Kim, A. F. Michael, and E. J. Yunis. 1975. C2 deficiency in man. Genetic relationship to a mixed lymphocyte reaction determinant (7a*). Immunogenetics. 2: 569-576.
42. Rubenstein, P., N. Suciu-Foca, J. F. Nicholson, M. Fotino, A. Molinaro, L. Harisiadis, M. A. Hardy, K. Reemtsma, and F. H. Allen, Jr. 1976. The HLA system in families of patients with juvenile diabetes mellitus. $J$. Exp. Med. 143: 1277-1282.

43. Dorf, M. E., J. H. Stimpfling, and B. Benacerraf. 1975. Requirement for two $\mathrm{H}-2$ complex Ir genes for the immune response to the L-Glu, L-Lys, L-Phe terpolymer. J. Exp. Med. 141: 1459-1463.

44. Dorf, M. E., P. H. Maurer, C. F. Merryman, and B. Benacerraf. 1976. Inclusion group systems and cis-trans effects in responses controlled by the two complementary Ir-GL $\phi$ genes. J. Exp. Med. 143: 898-896.

45. Démant, P., J. Capková, E. Hinzová, B. Vorácová. 1973. The role of the histocompatibility-2-linked $S s$-Slp region in the control of mouse complement. Proc. Natl. Acad. Sci. U.S.A. 70: 863-864.

46. Meo, T., T. Krasteff, and D. C. Shreffler. 1975. Immunochemical characterization of murine $\mathrm{H}$-2 controlled Ss (serum substance) protein through identification of its human homologue as the fourth component of complement. Proc. Natl. Acad. Sci. U.S.A. 72: 4536-4540.

47. Lachman, P. J., D. Grennan, A. Martin, and P. Demant. 1975. Identification of Ss protein as murine C4. Nature (Lond.). 258: 242-243.

48. Curman, B., L. Östberg, L. Sandberg, I. MalmhedenEriksson, G. Stålenheim, L. Rask, P. A. Peterson. 1975. H-2 linked Ss protein is C4 component of complement. Nature (Lond.). 258: 243-245.

49. Ferreira, A., and V. Nussenzweig. 1976. Control of C3 levels in mice during ontogeny by a gene in the central region of the $\mathrm{H}-2$ complex. Nature (Lond.). 260: 613-615. 